\title{
Reducing the impact of solar energy shortages on the wireless access network powered by a PV panel system and the power grid
}

\author{
Margot Deruyck *, Daniela Renga ${ }^{\dagger}$, Michela Meo ${ }^{\dagger}$, Luc Martens ${ }^{*}$, Wout Joseph * \\ ${ }^{*}$ Ghent University/iMinds - WAVES, Dept. of Information Technology, \\ Technologiepark-Zwijnaarde 15,B-9052 Ghent, Belgium, margot.deruyck@intec.ugent.be \\ $\dagger$ Dipartimento di Elettronica e Telecomunicazioni, Politecnico di Torino, Corso Duca degli Abruzzi 24, 10129 Torino, Italy
}

\begin{abstract}
In this study, the potential of applying different strategies to reduce the energy consumption of a wireless access network, powered by a photovoltaic panel system, during energy shortages is investigated. The goal is to reduce the amount of energy that should be bought from the traditional energy grid during a renewable energy shortage. Three different strategies are compared and the results are looking very promising. Depending on the strategy, up to $72 \%$ less energy should be bought compared to the fully operational network for a worst case scenario and a time period of 1 week. However, applying such a strategy has also its influence on the network performance. The influence on the user coverage is limited, with a reduction of $3 \%$ at maximum, but the capacity offered by the network decreases significantly with $51 \%$ up to even $71 \%$.
\end{abstract}

\section{INTRODUCTION}

Nowadays, a movement towards the use of renewable energy sources as provisioning system can be noticed. The reason for this is on the one hand the possible future depletion of our fossil fuels and on the other hand the increasing energy costs. Wireless cellular access networks, and more specific the base stations active in these networks, are large power consumers, with base stations accounting for up to $80 \%$ of energy consumption in $3 \mathrm{G}$ and LTE mobile networks [1]. The first studies about powering these base stations with renewable energy sources have recently appeared in literature [2]-[6] and several examples are already available of mobile networks where BSs are powered by solar and wind energy. The main driver to deploy such networks was first given by the need of dealing with the absence or unreliability of the traditional power grid, in some remote areas where the mobile service was to be provided. Nevertheless, current research in this scope strongly aims at investigating the promising role of renewable energy in mobile networks for reducing the grid energy consumption and the electricity costs. Furthermore, in the future, the wireless access network will become part of the smart grid, possibly off-grid with renewables as only energy sources [7]. One of the drawbacks of using renewable energy sources is that they are not able to offer the same supply continuity as currently been provided by fossil fuels or more traditional generators due to e.g., varying weather conditions. The different parts of the smart grid will have to align their energy need on each other [8]. So far, many studies have addressed the implementation of radio resource management techniques in mobile networks, based on sleeping strategies, to make them more energy efficient, but only few recent works (like [9], [10]) investigate the integration of energy-aware management of radio resources with the use of renewable energy sources in mobile networks, with the purpose of both reducing the grid energy consumption and complying with the typically intermittent availability of renewable energy.

In this paper, we investigate the potential of applying a strategy to reduce the energy consumed by the wireless access network when there is insufficient renewable energy available (not enough produced and/or stored). It is assumed that the network is connected to both a PV (Photovoltaic) generator and the traditional power grid. The purpose is to limit the amount of power that should be bought from the power grid. Different strategies are proposed and compared to the fully operational network. Furthermore, it is investigated if introducing a time window allows to save and store some energy in advance for a future predicted energy shortage.

The outline of this paper is as follows. Section II describes the methodology and the different strategies here considered. Section III presents the considered scenario and compares the results of the different strategies, whether or not taking into account a time window.

\section{Methodology}

To investigate the influence of these strategies on network performance, a deployment tool for wireless access networks is used and combined with predictions of the solar energy production. A worst case scenario is considered during 1 week for a small urban area with 8 macrocell base stations each supporting 4 microcell base stations. For each time slot, the algorithm determines if an energy shortage will occur (now or in the next few time slots if a time window is considered) and if so, it will apply the considered strategy (turning some or all microcell base stations off). Both the energy performance and the network performance will be compared for the different strategies by using the deployment tool.

\section{A. Scenario}

Fig. 1 shows the considered area of $0.3 \mathrm{~km}^{2}$ in a suburban environment. There are 8 macrocell base stations situated in this area (indicated as red squares in Fig. 1) supporting 
each 4 microcell base stations (represented as blue squares in Fig. 1). Just as an example, we have added the user locations as well in Fig. 1 (as yellow triangles). Note that the time slot starting at 5 p.m. of the first day is shown here, since it is the time slot with the highest amount of active users. As wireless technology, LTE (Long Term Evolution) Advanced is considered with a frequency of $2.6 \mathrm{GHz}$ and a channel bandwidth of $5 \mathrm{MHz}$ with only one transmitting and receiving antenna (i.e., SISO (Single Input Single Output)) for both the macrocell and the microcell base station. The other link budget parameters are assumed to be the same as in [12].

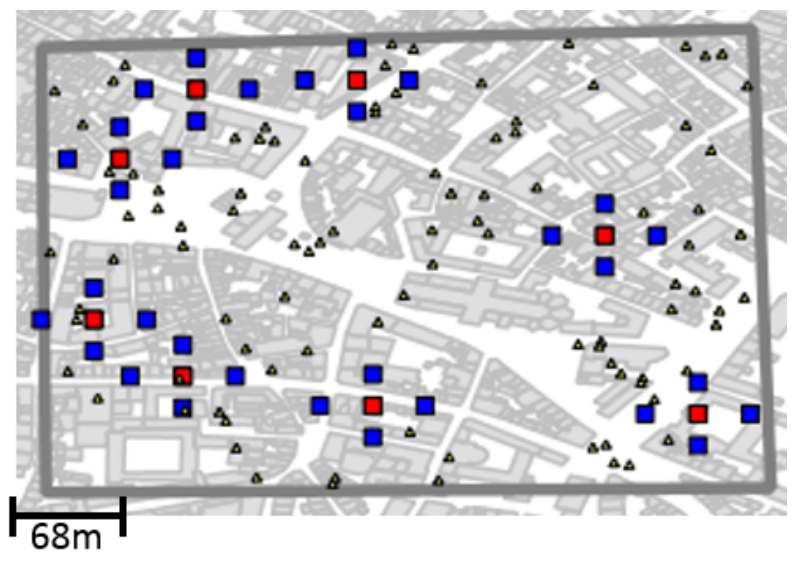

Fig. 1. Base station (red squares $=$ macrocell base station, blue square $=$ microcell base station) and users locations (yellow triangles) in the considered suburban area $\left(0.3 \mathrm{~km}^{2}\right)$ for the 5 p.m. time slot.

\section{B. Deployment tool: accounting for solar energy predictions}

To investigate the potential of different strategies to limit the amount of energy that should be bought, the capacitybased deployment tool of [11] is extended. The goal of this deployment tool is to develop a network with a minimal power consumption by taking into account the instantaneous bit rate required by the user. This optimization towards power consumption is obtained by selecting the most appropriate base station locations from a set of possible (existing) base station locations, fine tuning the input power of the base station's antenna and using sleep modes for those base stations that were not selected. Fig. 2 shows the flow diagram of the algorithm. For the simulations, different time slots (Fig. 2 Step 1) will be considered as the energy production by PV panels will fluctuate due to varying weather conditions. For each time slot (e.g., for each hour during one week), we will first determine how many users are active in the considered area (Fig. 2 Step 2). This will be based on the user predictions during the day. Once the number of active users is known, the network can be determined by using the algorithm proposed in [11] (Step 3). We assume here that each active macrocell base station will support four different microcell base stations. Furthermore, a microcell base station can not be awakened when the macrocell base station is still in sleep mode.

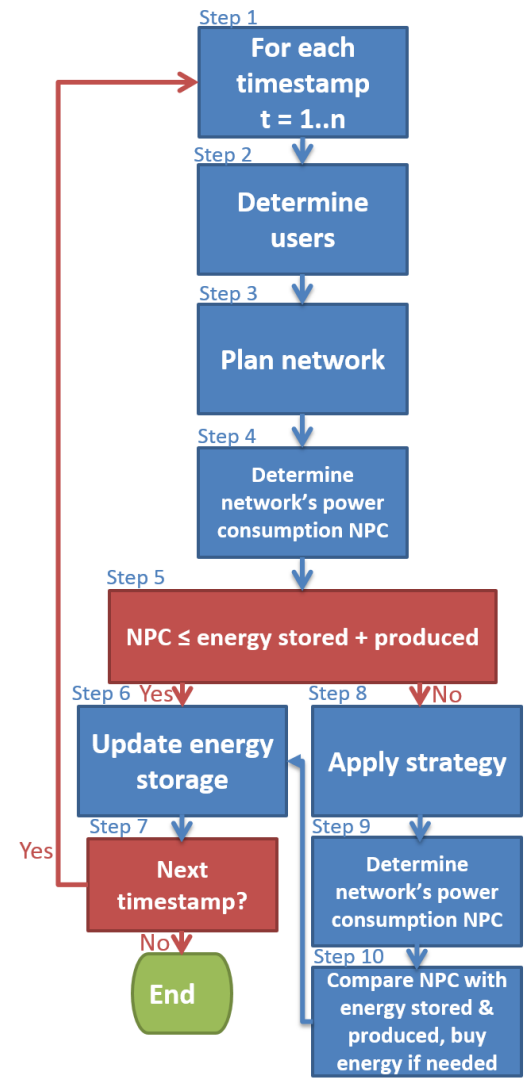

Fig. 2. Flow diagram of the algorithm $(\mathrm{NPC}=$ power consumption of the network).

Once the network is determined, the power consumption is calculated (Step 4, NPC = power consumption of the network). To this end, the power consumption model for the macrocell and the microcell base station proposed in [12] will be considered. The network's power consumption will then be compared with the amount of energy available in the energy storage and produced by the PV panels (Step 5). To model the energy produced by the PV panels, the online tool PVWatts Calculator is considered [13]. If the network consumes less energy than the amount of energy stored and produced, no energy reducing strategy should be applied. We only need to update the energy storage (Step 6) by enhancing it with the amount of produced energy that is not consumed (in case the energy production is sufficient to feed the network) or by diminishing it (in case the energy production alone is not sufficient enough). Thereafter, we can proceed with the next time slot (Step 7) if there are any left to investigate. If the network consumes more energy than the amount of energy stored and produced, an energy reducing strategy is applied (Step 8). Five different strategies are considered and will be discussed in the next subsection. Applying an energy reducing strategy will of course change the network's power consumption (Step 9) and we have to check again if the amount of energy stored and produced is sufficient or not (Step 10). In case it is insufficient, the amount of energy that 
can not be provided by the PV panels and the storage needs to be bought from the traditional grid (Step 10). Whether or not we should buy energy, the energy storage needs to be updated (Step 6) and the algorithm can proceed with the next time slot if there are any left (Step 7), otherwise the simulation will stop.

\section{Energy production and storage}

For the energy production, 1 week is considered. Predictions for the period of 3 January until 9 January for the area of Turin, Italy will be used [13]. Note that this is a period with limited sunshine and is assumed as a worst case scenario. Our simulations are starting on 3 January at midnight. A time slot of $1 \mathrm{~h}$ is considered and a time window of $5 \mathrm{~h}$ when the last two strategies of Section II-D are applied.

For the 8 macrocell base stations (each supporting 4 microcell base stations), a PV panel size of $100 \mathrm{kWp}$ is considered, which results in a PV panel size of $12.5 \mathrm{kWp}$ for each macrocell base station. According to [3], [4], [5], where the same location is considered, a PV panel with size ranging between 9.3 and $16.6 \mathrm{kWp}$, combined with a proper battery dimensioning, allows to power a macrocell base station with locally produced renewable energy and no need for energy taken from the traditional grid. With a $16.6 \mathrm{kWp} \mathrm{PV} \mathrm{system,}$ more than $60 \mathrm{kWh}$ of storage would be required in order to make the network completely independent from the traditional grid, but a smaller storage can be assumed if we accept that the network may not necessarily be completely off-grid.

A battery size of $50 \mathrm{kWh}$ (actually $71-100 \mathrm{kWh}$, due to a maximum Depth of Discharge of $70 \%$ or $50 \%$ respectively, to ensure maximum battery life) has been assumed. A similar storage size is too low to make the system completely independent from the traditional grid; however, this is not the main purpose of this work, whose aim is rather investigating the impact of locally produced renewable energy in reducing the consumption from the grid, by exploiting renewable energy at peak time and by resource management when the traffic, hence the energy demand, is lower (especially by night, when the renewable energy is not available). In relation to the PV system area, around $4.9 \mathrm{~m}^{2}$ per $\mathrm{kWp}$ can be assumed, for a total area of $490 \mathrm{~m}^{2}$ [3]. A distributed physical implementation of the PV and storage system is assumed, assigning to each of the 8 base station cluster locations a PV panel with capacity of $12.5 \mathrm{kWp}$ and a battery with maximum depth of charge of $6.25 \mathrm{kWh}$. However, for the algorithm, the PV panels and batteries are treated as they were a unique PV system, grouping all the PV panels, and a unique storage, made up by all the single batteries. Therefore, the extra amount of renewable energy produced by each PV panel can be freely stored considering the whole available battery capacity, while any base station can draw the available stored energy from the unique battery without any constraints. A central controller is assumed to handle the centralized management of this physically distributed system and the needed information exchange for proper system operation. At the start of the simulation, the battery is assumed to be completely full. Furthermore, we are neglecting storage losses occurring both during battery charge and discharge and for energy transfer/transmission.

\section{Strategies}

Five different strategies are here considered:

1) No action at all: no energy will be saved with this strategy of course, but it is important to consider it as it represents the current situation. This will be considered as the reference scenario and also referred to as the fully operational network.

2) Switching off all microcell base stations: when there is not enough available energy, all microcell base stations will be switched off in order to save energy. All users connected to these microcell base stations will be reconnected to a macrocell base station if possible.

3) Switching off 1 to 4 microcell base stations: in this case, only 1 to 4 microcell base stations per macrocell base station will be switched off depending on the amount of energy that should be bought when no action is taken. Note that if multiple microcell base stations of a particular macrocell base station need to be switched off, this will happen in the order of the number of connected users. The microcell base station serving the lowest number of users will be switched off first. In this way, less users need to be reconnected to another microcell or macrocell base station.

4) Switching off all microcell base stations + time window $t_{w}$ : when the algorithm notices that energy needs to be bought in the next $t_{w}$ time slots, it will already take action for the current time slot. The strategy here applied is the same as the second strategy.

5) Switching off 1 to 4 microcell base stations + time window $t_{w}$ : same strategy as the third strategy. Only here, the strategy will be applied when the algorithm notices that energy should be bought in the next $t_{w}$ time slots.

\section{RESULTS}

\section{A. Evolution of the energy consumption, production, and storage during the week}

As mentioned above, simulations have been performed for one week during the month of January (worst case scenario). Fig. 3 shows the evolution of the network's energy consumption (in case no action is taken when an energy shortage is occurring), the renewable energy production by the PV panel system, and the energy storage. The blue (dashed) line in Fig. 3 represents the energy consumed by the network. As confirmed by [11], the network's power consumption is related to the number of active users. The highest power consumption is everyday obtained around 5 p.m. (e.g., $22.8 \mathrm{~kW}$ for time slot 17) and the lowest around 4 a.m. (e.g., $8.3 \mathrm{~kW}$ for time slot 4 ). The higher the number of active users, the more base stations that are being awake, and thus the higher the network's power consumption.

The cyan (full) line in Fig. 3 shows the production of renewable energy by the PV panel system. The amount of 


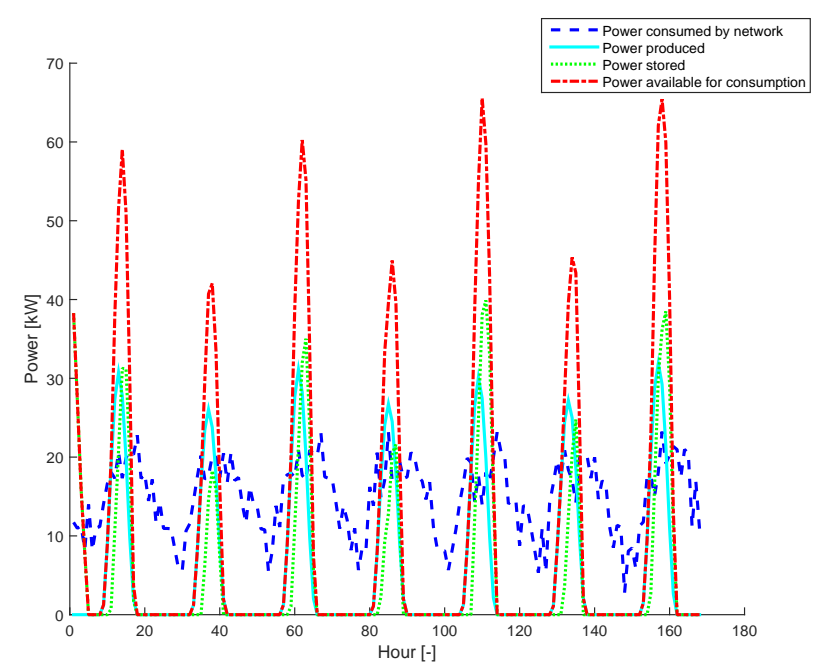

Fig. 3. Evolution of the network's energy consumption, the renewable energy production by the PV panel system, and the energy storage when no action is taken in case of (renewable) energy shortage.

energy that is produced is limited to only a few hours a day (around midday). As mentioned above, the first week of January is simulated which is a worst case scenario with limited amount of sunshine during the day.

The green (dotted) line in Fig. 3 represents the amount of energy that is stored on the battery. During the day, when there is enough energy produced (cyan line is above the blue line), the battery is charged, while in the early evening the battery starts to discharge as less to no energy is produced (cyan line is below blue line). The battery is completely discharged during the night.

Finally, the red (dashed-dotted) line shows the amount of renewable energy that is available for consumption. This corresponds with the amount of energy produced plus the amount of energy stored on the battery. When the red line drops below the blue line, there is not enough renewable energy available and energy should be bought from the traditional grid. When no action is taken to reduce the network's power consumption, we should buy energy for $73 \%$ of the time.

\section{B. Comparing strategies without using a time window}

Table I compares the results for the different strategies described in Section II-D without taking into account a time window. Regarding the energy performance of the different strategies, two parameters are considered: the total energy consumed by the network and the amount of energy that should be bought from the traditional grid. Based on the results of Table I, we can conclude that it is certainly useful to apply a strategy in case of an energy shortage. When switching off all microcell base stations in case of insufficient renewable energy, 52.9\% less energy should be bought compared to no action at all $(755.9 \mathrm{~kW}$ bought versus 1605.2 kW, Table I).

\section{1) Bought energy:}

When switching off 1 to 4 microcell base stations, $50.4 \%$ less energy should be bought compared to no action at all (796.7 kW versus $1605.2 \mathrm{~kW}$, Table I). Slightly more energy should be bought with the latter strategy than when we switch off all microcell base stations because this strategy uses almost all available renewable energy by keeping as much microcell base stations active as possible, thus saving less energy for future energy shortages. This is also the reason why this strategy consumes slightly more energy than when we switch off all microcell base stations $(1723.8 \mathrm{~kW}$ versus $1699.3 \mathrm{~kW}$, Table I). However, both strategies clearly reduce the amount of consumed energy by $32.7 \%$ and $31.7 \%$, respectively, compared to when we do not apply any strategy at all $(1699.3 \mathrm{~kW}$ and $1723.8 \mathrm{~kW}$ respectively versus $2525.0 \mathrm{~kW}$, Table I).

TABLE I

ENERGY AND NETWORK PERFORMANCE OF THE THREE DIFFERENT STRATEGIES WITHOUT A TIME WINDOW IN CASE OF (RENEWABLE) ENERGY SHORTAGE.

\begin{tabular}{|l|c|c|c|c|c|}
\hline & \multicolumn{2}{|c|}{ Energy performance } & \multicolumn{3}{|c|}{ Network performance } \\
\hline & $\begin{array}{c}\text { Total } \\
\text { energy } \\
\text { consumed } \\
{[\mathrm{kW}]}\end{array}$ & $\begin{array}{c}\text { Total } \\
\text { energy } \\
\text { bought } \\
{[\mathrm{kW}]}\end{array}$ & $\begin{array}{c}\text { Average } \\
\text { geometrical } \\
\text { coverage } \\
{[\%]}\end{array}$ & $\begin{array}{c}\text { Average } \\
\text { user } \\
\text { coverage } \\
{[\%]}\end{array}$ & $\begin{array}{c}\text { Average } \\
\text { network } \\
\text { capacity } \\
{[\mathrm{Mbps}]}\end{array}$ \\
\hline No action & 2525.0 & 1605.2 & 85.4 & 96.7 & 444.2 \\
\hline $\begin{array}{l}\text { all microcell } \\
\text { BSs off }\end{array}$ & 1699.3 & 755.9 & 84.8 & 94.7 & 208.7 \\
\hline $\begin{array}{l}\text { 1 to 4 microcell } \\
\text { BSs off }\end{array}$ & 1723.8 & 796.7 & 85.7 & 94.9 & 215.8 \\
\hline
\end{tabular}

\section{2) Coverage:}

Looking at the network performance, the geometrical coverage, the user coverage, and the network capacity is considered, all averaged over the 1 week simulation. For the average geometrical coverage, the difference between the different strategies is very limited $(85.4 \%$ for no action, $84.8 \%$ when switching off all microcell base stations, and $85.7 \%$ when switching off 1 to 4 microcell base stations, Table I). The main reason is because the algorithm described above does not focus on maximizing the geometrical coverage at all, but on maximizing the user coverage.

The lowest average user coverage is obtained when we switch off all microcell base stations in case of an energy shortage $(94.7 \%$ versus $96.7 \%$ for no action and $94.9 \%$ when switching 1 to 4 microcell base stations off, Table I). As all microcell base stations are switched off, the options to reconnect each user which was connected to a microcell base station before are very limited, especially when the capacity of the macrocell base stations is completely used, resulting thus in disconnecting some users and thus a lower average user coverage. The difference in average user coverage is not significant between the strategy where we switch off all microcell base stations and the strategy where we switch off 1 to 4 microcell base stations (only $0.2 \%$ lower when switching off all users, Table I). This is due to the fact that the number of time slots where we can switch off less than 4 
microcell base stations are limited.

3) Capacity:

The highest average network capacity is of course obtained when no base stations are switched off. When switching off all microcell base stations, the average network capacity is reduced by $53.0 \%$ compared to no action at all (208.7 Mbps versus $444.2 \mathrm{Mbps}$, Table I). This is of course due to the fact that all microcell base stations are switched off, thus reducing the available network capacity significantly. When switching off 1 to 4 microcell base stations, the average network capacity is reduced by $51.4 \%$ compared to no action. The average network capacity is slightly less reduced than when switching off all microcell base stations due to these few time slots where it is possible to switch only 1 to 3 microcell base stations.

\section{Influence of taking into account a time window}

In the previous section, only the current time slot was taken into account, while future (renewable) energy shortages are not accounted for. In this section, we investigate if it is interesting to save some energy in advance for future energy shortages, and further reduce the amount of energy that should be bought from the traditional energy grid. Table II shows the results for the same three strategies while assuming a time window of $5 \mathrm{~h}$. This means that if a (renewable) energy shortage is noticed in the next 5 hours, the algorithm will already take action for the current time slot. There is of course no difference in the results between Table I and Table II for the first strategy as no action is taken in case of a shortage. Furthermore, the same difference in energy performance and network performance can be noticed between the other strategies. The same reasons as mentioned above apply here and we refer the reader to Section III-B for more information.

TABLE II

ENERGY AND NETWORK PERFORMANCE OF THE THREE DIFFERENT STRATEGIES WITH A 5H TIME WINDOW IN CASE OF (RENEWABLE) ENERGY SHORTAGE.

\begin{tabular}{|l|c|c|c|c|c|}
\hline & \multicolumn{2}{|c|}{ Energy performance } & \multicolumn{3}{|c|}{ Network performance } \\
\hline & $\begin{array}{c}\text { Total } \\
\text { energy } \\
\text { consumed } \\
{[\mathrm{kW}]}\end{array}$ & $\begin{array}{c}\text { Total } \\
\text { energy } \\
\text { bought } \\
{[\mathrm{kW}]}\end{array}$ & $\begin{array}{c}\text { Average } \\
\text { geometrical } \\
\text { coverage } \\
{[\%]}\end{array}$ & $\begin{array}{c}\text { Average } \\
\text { user } \\
\text { coverage } \\
{[\%]}\end{array}$ & $\begin{array}{c}\text { Average } \\
\text { network } \\
\text { capacity } \\
{[\mathrm{Mbps}]}\end{array}$ \\
\hline No action & 2525.0 & 1605.2 & 85.4 & 96.7 & 444.2 \\
\hline $\begin{array}{l}\text { all microcell } \\
\text { BSs off }\end{array}$ & 1403.9 & 448.2 & 85.0 & 93.7 & 129.2 \\
\hline $\begin{array}{l}1 \text { to } 4 \text { microcell } \\
\text { BSs off }\end{array}$ & 1544.7 & 615.8 & 85.2 & 94.1 & 163.8 \\
\hline
\end{tabular}

\section{1) Bought energy:}

Looking at the two strategies where action is taken in case of insufficient renewable energy, a further reduction is noticed in the amount of energy that should be bought from the traditional grid. When all microcell base stations are switched off, an extra reduction of $40.7 \%$ is noticed $(448.2 \mathrm{~kW}$ for $5 \mathrm{~h}$ time window versus $755.9 \mathrm{~kW}$ for no time window, Tables I \& II), which is in total $72.1 \%$ less energy that should be bought compared to the case where no action is taken (615.8.2 kW versus $1605.2 \mathrm{~kW}$, Tables I \& II). When only
1 to 4 microcell base stations are switched off, a further reduction of $22.7 \%$ is noticed $(615.8 \mathrm{~kW}$ for $5 \mathrm{~h}$ time window versus $796.7 \mathrm{~kW}$ for no time window, Tables I \& II), which is a total reduction of $54.4 \%$ compared to no action at all (615.8 kW versus $1605.2 \mathrm{~kW}$, Tables I \& II). This results also in a further reduction of the overall network's power consumption. An extra $17.4 \%$ and $10.4 \%$ reduction is found when applying a $5 \mathrm{~h}$ time window for the strategy where we switch off all the microcell base stations and where we switch off 1 to 4 microcell base stations respectively $(1403.9 \mathrm{~kW}$ versus $1699.3 \mathrm{~kW}$ and $1544.7 \mathrm{~kW}$ versus $1723.8 \mathrm{~kW}$, respectively, Tables I \& II). Compared to the strategy where no action is taken, a total reduction of $50.1 \%$ and $42.1 \%$ is found when switching off all microcell base stations, respectively 1 to 4 microcell base stations (1403.9 $\mathrm{kW}$ and $1544.7 \mathrm{~kW}$ respectively versus $2525.0 \mathrm{~kW}$, Tables I \& II).

\section{2) Coverage:}

Considering the network performance, the average geometrical coverage does not significant differ between the results obtained with no time window and a $5 \mathrm{~h}$ time window. As mentioned above, the algorithm focuses on optimizing the user coverage, and not the geometrical coverage. The average user coverage and the average network capacity is further reduced for both strategies where we switch off microcell base stations. Compared to the case with no time window, the average user coverage decreases with $1 \%$ when all microcell base stations are switched off $(93.7 \%$ for no time window versus $94.7 \%$ for a $5 \mathrm{~h}$ time window), and $0.8 \%$ when switching off 1 to 4 microcell base stations $(94.1 \%$ versus $94.9 \%$ ). This is due to the fact that when a $5 \mathrm{~h}$ time window is assumed, the power reducing strategy is used during more time slots to save and store some energy in advance for future shortages, thus reducing the average user coverage.

\section{3) Capacity:}

The same considerations made above apply to the reduction in average network capacity. When switching off all microcell base stations, the average network capacity is further reduced by $38.1 \%$ compared when no time window is taken into account (129.2 Mbps versus 208.7 Mbps, Tables I \& II), resulting in a total decrease of $70.9 \%$ compared when the network is fully operational during all time slots (129.2 Mbps versus 444.2 Mbps, Tables I \& II). When switching off 1 to 4 microcell base stations, the average network capacity is decreased with $24.1 \%$ compared when no time window is considered (163.8 Mbps versus 215.8 Mbps, Tables I \& II), and a total reduction of $63.1 \%$ is found compared to the fully operational network (163.8 Mbps versus 444.2 Mbps, Tables I \& II).

\section{Conclusion}

In this paper, the potential benefits of applying strategies to reduce the energy consumption of a network powered by a PV panel system during energy shortage is investigated. The goal is to reduce the amount of energy that should be bought from 
the traditional energy grid when a renewable energy shortage occurs. The network consists of a mixture of macrocell and microcell base stations. Three different strategies, as well as the influence of time window whereby a strategy is applied in advance to save energy for a future expected energy shortage, are studied.

In general, the results show that applying such a strategy is very promising. About 50\% less energy should be bought from the traditional grid for a worst case scenario and a time period of 1 week. Using such a strategy has of course its repercussions on the network performance. The influence on the average user coverage is limited as it decreases with approximately $2 \%$. However, the average capacity offered by the network drops by 51 to $54 \%$ (depending on which strategy is considered). When considering a $5 \mathrm{~h}$ time window, 54 to $72 \%$ less energy should be bought compared to the fully operational network. The user coverage decreases with 2.6 to $3 \%$, while the network capacity even drops with 63 up to $71 \%$. If the energy performance (i.e., minimizing the amount of bought energy from the traditional grid) is the primary goal, the strategy which switches off all microcell base stations is the most appropriate to use, preferably in combination with the use of a time window to account for future shortages. If one finds it important to preserve the network performance as well, the strategy whereby the number of switched off microcell base stations depends on the amount of energy that needs to be saved is the most appropriate one.

As future work, an extensive sensitivity analysis should be performed in order to identify the influence of the different parameters such as the energy production system size, the energy storage size, and the time window on the results. Furthermore, more advanced strategies should be developed as well.

\section{ACKNOWLEDGMENT}

Margot Deruyck is supported by a postdoctoral grant from the Special Research Fund (BOF) of Ghent University (Belgium) and received a travel grant from FWO (Research Foundation Flanders) to perform research at the Politecnico di Torino.

\section{REFERENCES}

[1] M. Etoh, T. Ohya, Y. Nakayama, "Energy Consumption Issues on Mobile Network Systems", Applications and the Internet, 2008. SAINT 2008. International Symposium on, pp. 365-368, 2008.

[2] H. Al Haj Hassan, L. Nuaymi, A. Pelov, "Renewable energy in cellular networks: A survey" Online Conference on Green Communications (GreenCom), 2013 IEEE, pp.1-7, 29-31 Oct. 2013.

[3] M.A. Marsan, G. Bucalo, A. Di Caro, M. Meo, Y. Zhang, 'Towards zero grid electricity networking: Powering BSs with renewable energy sources", IEEE International Conference on Communications Workshops (ICC), pp. 596-601, 2013.

[4] M.A. Marsan, R. Gerboni, J.G. Iglesias, M. Meo, Y. Zhang, ”Optimizing the Power Supply of a Macro BS Connected to a PV Panel and the Power Grid", GTTI meeting (Gruppo Telecommunicazioni e Tecnologie dell'Informazione, 2014.

[5] M. Meo, Y. Zhang, R. Gerboni, M.A. Marsan, "Dimensioning the power supply of a LTE macro BS connected to a PV panel and the power grid", IEEE International Conference on Communications (ICC), pp. 178-184, 2015.
[6] H. Tao, N. Ansari, ”On Optimizing Green Energy Utilization for Cellular Networks with Hybrid Energy Supplies" in Wireless Communications, IEEE Transactions on, vol.12, no.8, pp.3872-3882, August 2013.

[7] X. Fang, S. Misra, G. Xue, D. Yang, "Smart Grid - The New and Improved Power Grid: A Survey" in Communications Surveys \& Tutorials, IEEE, vol.14, no.4, pp.944-980, Fourth Quarter 2012.

[8] M.S. Obaidat, A. Anpalagan, I. Woungang, "Handbook of Green Information and Communication Systems", Academic press, 2013, ISBN: 9780-1241-5844-3.

[9] H. Ghazzai, E. Yaacoub, M.-S. Alouini, A. Abu-Dayya, "Optimized green operation of LTE networks in the presence of multiple electricity providers" in Globecom Workshops (GC Wkshps), 2012 IEEE, pp.664669, 3-7 Dec. 2012.

[10] H. Ghazzai, E. Yaacoub, M.-S. Alouini, A. Abu-Dayya, "Performance of Green LTE Networks Powered by the Smart Grid with Time Varying User Density" in Vehicular Technology Conference (VTC Fall), 2013 IEEE 78th , pp.1-6, 2-5 Sept. 2013.

[11] M. Deruyck, W. Joseph, E. Tanghe, L. Martens, "Reducing the power consumption in LTE-Advanced wireless access networks by a capacity based deployment tool", Radio Science, Vol. 49, No. 9, pp. 777-787, 2014.

[12] M. Deruyck, W. Joseph, L. Martens, "Power consumption model for macrocell and microcell base stations", Transactions on Emerging Telecommunications Technologies, Vol. 25, No. 3, pp. 320-333, 2014.

[13] http://pvwatts.nrel.gov/pvwatts.php, last accessed: February 2015. 